\title{
Paraneoplastic autoimmune multi- organ syndrome is a distinct entity from traditional pemphigus subtypes
}

\section{Kyle T. Amber}

I commend the authors of the Primer on pemphigus (Pemphigus. Nat. Rev. Dis. Primers 3, $17026(2017))^{1}$ for their detailed review of this disease, which is an excellent and highly defined model to study human autoimmunity. In the Primer, the authors included paraneoplastic pemphigus (PNP) as a subtype of pemphigus; however, it should be noted that a different classification of PNP has been described, based on differences in the underlying pathophysiological mechanisms. The term PNP was coined after identifying shared serological immunopathological features with pemphigus ${ }^{2}$. Clinically, PNP has a wide spectrum of morphological presentations: it can mimic erythema multiforme, lichen planus pemphigoides, bullous pemphigoid, graft-versus-host disease or pemphigus $^{3}$. In fact, a lichenoid presentation has more commonly been described than a pemphigus presentation ${ }^{4}$. Furthermore, the clinical course of PNP can be distinct from that of other pemphigus subtypes like pemphigus vulgaris or pemphigus foliaceus. For example, PNP does not always respond to treatments that usually resolve pemphigus, the spectrum of autoantibodies in patients with PNP is different from that observed in pemphigus vulgaris or pemphigus foliaceus with the presence of non-skin autoantibodies, and PNP by definition is associated with malignancy or Castleman disease. For example, in patients with Castleman disease and a PNP phenotype, removal of the tumours resulted in the resolution of PNP ${ }^{5}$. Thus, PNP would be more appropriately considered a single pemphigus-like mucocutaneous phenotype of paraneoplastic autoimmune multi-organ syndrome (PAMS) ${ }^{6}$. Here, I present some evidence supporting this classification.

Clinically, PNP has many distinctions from pemphigus vulgaris and pemphigus foliaceus, supporting the notion that PNP is an unrelated condition. The cutaneous manifestations of pemphigus subtypes frequently involve the scalp and usually do not present on palms and soles, whereas, as the authors note, in PNP there is "involvement of palms and soles but not the scalp." Indeed, the involvement of the palms and soles is common in pemphigoid diseases ${ }^{7}$. When PNP affects the oral mucosa, the PNP is far more diffuse and severe than that observed in pemphigus vulgaris, resulting in erosive stomatitis ${ }^{8}$.

In marked contrast to pemphigus vulgaris and pemphigus foliaceus, in which mortality does not exceed $10 \%{ }^{9}$, a fatal outcome is observed in $90 \%$ of patients with $\mathrm{PNP}^{10}$. The authors of the Primer state that this mortality could be due to underlying malignancy, infection or bronchiolitis obliterans (BO), which could result from fibrotic changes in the bronchioles induced by desmoglein 3 -specific $\mathrm{CD} 4^{+}$ $\mathrm{T}$ cells that target desmoglein 3 -expressing cells in squamous metaplasia in the lung ${ }^{11}$. However, although metaplasia is found in the affected pulmonary epithelial lining of patients with PNP with pulmonary involvement, metaplastic human bronchial epithelia do not express desmoglein $3^{9}$. Thus, fibrosis and $\mathrm{BO}$ are probably mediated by $\mathrm{T}$ cells and autoantibodies against epiplakin, which is expressed by human pulmonary epithelia ${ }^{6,12}$.

Similarly, fibrosis is not the hallmark of pemphigus vulgaris or pemphigus foliaceus. The presence of anti-desmoglein 3 autoantibody-induced pulmonary fibrosis in mice is not consistent with humans with pemphigus vulgaris, who do not develop pulmonary fibrosis. As desmoglein 3 is present at other sites, namely the oesophagus, pharynx, anal canal and vagina, it is unclear why fibrosis in patients with PNP only occurs in the lung. Whether lung-specific metaplasia precipitates
BO remains unclear. Indeed, sloughing of bronchial epithelial cells can contribute to occlusion of the small airways, providing a potential mechanism for the respiratory failure in patients with $\mathrm{PNP}^{6}$. Finally, metaplasia is not typically observed in autoimmune conditions but is a feature of precancerous or cancer pathology, which may account for the over-representation of Castleman disease in patients with PNP and $\mathrm{BO}^{13}$.

Kyle T. Amber

Department of Dermatology, University of California Irvine Health, Irvine, CA, USA. e-mail: KAmber@UCl.edu

doi: $10.1038 /$ nrdp. 2018.12 Published online 22 Feb 2018

Kasperkiewicz, M. et al. Pemphigus. Nat. Rev. Dis. Primers 3, 17026 (2017)

2. Anhalt, G. J. et al. Paraneoplastic pemphigus. An autoimmune mucocutaneous disease associated with neoplasia. N. Engl. J. Med. 323, 1729-1735 (1990).

3. Frew, J. W. \& Murrell, D. F. Paraneoplastic pemphigus (paraneoplastic autoimmune multiorgan syndrome): clinical presentations and pathogenesis. Dermatol. Clin. 29, 419-425 (2011).

4. Mimouni, D. et al. Paraneoplastic pemphigus in children and adolescents. Br. J. Dermatol. 147, 725-732 (2002)

5. Fang, Y. et al. A critical role of surgery in the treatment for paraneoplastic pemphigus caused by localized Castleman's disease. Med. Oncol. 27, 907-911 (2010).

6. Nguyen, V. T. et al. Classification, clinical manifestations, and immunopathological mechanisms of the epithelial variant of paraneoplastic autoimmune multiorgan syndrome: a reappraisal of paraneoplastic pemphigus. Arch. Dermatol. 137, 193-206 (2001).

7. Schmidt, E. \& Zillikens, D. Pemphigoid diseases Lancet 381, 320-332 (2013).

8. Czernik, A., Camilleri, M., Pittelkow, M. R. \& Grando, S. A. Paraneoplastic autoimmune multiorgan syndrome: 20 years after. Int. J. Dermatol. 50, 905-914 (2011).

9. Kridin, K., Zelber-Sagi, S. \& Bergman, R. Mortality and cause of death in patients with pemphigus. Acta Derm. Venereol. 97, 607-611 (2017).

10. Nousari, H. C. et al. The mechanism of respiratory failure in paraneoplastic pemphigus. N. Engl. J. Med. 340, 1406-1410 (1999).

11. Hata, T. et al. Ectopic expression of epidermal antigens renders the lung a target organ in paraneoplastic pemphigus. J. Immunol. 191, 83-90 (2013).

12. Tsuchisaka, A. et al. Epiplakin is a paraneoplastic pemphigus autoantigen and related to bronchiolitis obliterans in Japanese patients. J. Invest. Dermatol. 136, 399-408 (2016).

13. Lee, J., Bloom, R. \& Amber, K. T. A systematic review of patients with mucocutaneous and respiratory complications in paraneoplastic autoimmune multiorgan syndrome: Castleman's disease is the predominant malignancy. Lung 193, 593-596 (2015).

Competing interests

The author declares no competing interests.

How to cite this article

Amber, K.T. Paraneoplastic autoimmune multi-organ syndrome is a distinct entity from traditional pemphigus subtypes. Nat. Rev. Dis. Primers. 4, 18012 (2018). 